\title{
Simulation of MQC code of SAC/Optical CDMA and WDM hybrid overlay system
}

\author{
Isaac A. M. Ashour ${ }^{\# 1}$, Student Member, IEEE, Hossam M. H. Shalaby ${ }^{* 2}$, Senior Member, IEEE, Sahbudin \\ Shaari $^{\# 3}$, Member, IEEE and P Susthitha Menon ${ }^{\# 4}$, Member, IEEE \\ ${ }^{\#}$ Institute of Microengineering and Nanoelectronics (IMEN), Universiti Kebangsaan Malaysia \\ 43600 UKM Bangi, Selangor, Malaysia \\ 1.isaacash@eng.ukm.my \\ ${ }^{3}$ sahbudin@eng.ukm.my \\ ${ }^{4}$ susi@eng.ukm.my \\ *Department of Electronics and Communications Engineering, School of Electronics, Communications, and Computer \\ Engineering, Egypt-Japan University of Science and Technology (E-JUST), Alexandria 21934, Egypt \\ ${ }^{2}$ hossam.shalaby@ejust.edu.eg
}

\begin{abstract}
A hybrid WDM and SAC/Optical CDMA System is proposed to enhance the data network security and to exploit the capacity of an optical system. Code pulses of SAC/Optical CDMA are overlaid onto a multichannel WDM system. Modified Quadratic Congruence (MQC) code is as the signature address code for SAC/Optical CDMA. In general, OCDMA improves service availability for data of a network. With the use of WDM channels in a hybrid scheme, an eavesdropper faces another challenge for intercepting and decoding the coded signal. The interference that is the main factor limits the performance of the proposed system. So that, the notch filters and APD photo detectors instead of PIN photo detectors are used in order to improve the SAC/OCDMA performance. A simulation is presented as a demonstration of the concept.
\end{abstract}

Keywords- Optical fiber communications, optical networks, optical code division multiple access (OCDMA), spectral amplitude coding (SAC), wavelength division multiplexing (WDM), Modified Quadratic Congruence (MQC).

\section{INTRODUCTION}

A security system for optical communication signals is getting more attractive concerns for some applications such as military networks and enterprise networks. Optical CDMA can be overlaid onto the existing WDM networks in order to expand the transmission capacity and add some additional functions in the networks such as increasing the levels of security. Spectral amplitude coding for optical code-division multiple-access (SAC/OCDMA) scheme yields a significant reduction in the effects of multiple access interference (MAI) and therefore provides an attractive solution for burst and asynchronous environments [1].

Many codes have been proposed for the SAC/OCDMA systems, e.g., Hadamard, m-sequences, Balanced Incomplete Block Design (BIBD), Modified Frequency Hopping (MFH), and Modified Quadratic Convergence (MQC). Modified quadratic congruence (MQC) code [2] is an effective code that can suppress the effects of intensity noise. This code defined as series $\left(p^{2}+p, p+1,1\right)$ codes for each prime number $p$ based on the quadratic congruence (QC) codes.

Recently the hybrid WDM/OCDMA system has been proposed for network security and demonstrations of WDM/OCDMA transmission have been performed [36 ]. These studies were focused on hybrid WDM and spectral-phase-coding (SPE)/OCDMA networks or hybrid WDM and wavelength-hopping time-spreading (WHTS) /OCDMA networks. Some other studies were concentrated on hybrid WDM and SAC/OCDMA schemes [1, 7-10] without using covertness of signal transmission. In other words, the SAC/OCDMA signals have not been overlaid with WDM signals in the same spectral region. Some other researchers $[11,12]$ proposed techniques using hybrid SAC/OCDMAoverlay WDM system, but complex codes such as frequency hopping have been adopted. However, the previous studies have not evaluated the performance of a hybrid MQC code of SAC/OCDMA - WDM overlay system.

In this paper, an enhancement of data network security using hybrid WDM and SAC/ OCDMA system is proposed. The MQC code of SAC/OCDMA-overlay WDM channels is used. The low complexity receiver includes notch filters for the hybrid system to suppress 
WDM interference for detection of optical broadband CDMA signals. In this paper we evaluate the comparison of hybrid and non-hybrid performance by simulation. In the following section we give a brief description of our proposed system, followed by the simulation setup, then results and discussion. Finally the conclusion of the paper is provided. It should be noted that we have also shown by simulation that the eavesdropper signal can be detected.

\section{SYSTEM DESCRIPTION}

In a hybrid WDM-OCDMA system, the network traffic consists of narrow-band WDM signals overlaid with optical broad-band CDMA signals in the same spectral region. The main idea in the proposed system is to enhance the security of the SAC/OCDMA by the hybrid scheme. The main purpose for this is to eliminate or attenuate the WDM interferences at the receiver. By using notch filters, WDM signals are attenuated properly. In fact a notch filter is a simplest technique among other techniques used for optical-carrier suppression [13]. Our SAC/OCDMA with MQC code consists of a single LED source and point-to-point transmission channels modulated at $622 \mathrm{Mbps}$ data rate, as shown in Fig. 1. MQC code families $\left(p^{2}+p, p+1,1\right)$ have properties that are mentioned in [2], where $\left(p^{2}+p\right)$ are elements for each code sequence divided into $(p+1)$ groups where each of them have one " 1 " and (p-1)" 0 "s, and $\lambda$ is its in-phase cross correlation equal to 1 between any two different sequences. A MQC code could be denoted by $(\mathrm{N}, \omega, \lambda)$, where $\mathrm{N}$ is the number of elements of the code sequence and $\omega$ is the code weight. The number of users $K$ equals $\mathrm{p}^{2}$. Table 1 shows the code sequences for parameters $\mathrm{p}=3, \mathrm{~N}=12$, and the selective wavelengths. The public WDM system setup consists of $\mathrm{CW}$ laser sources for some channels that are modulated at $2.5 \mathrm{Gbps}$ for each user. The two systems are using ON-OFF keying modulation and are combined together over a single fiber link. At the receiver side of the system, the WDM receiver consists of a narrowband-pass filter followed by $\mathrm{O} / \mathrm{E}$ conversion.

The SAC/CDMA receiver is based on the well known balanced receiver with notch filters designed for the hybrid system and PIN or APD photo detectors. It is worth mentioning that the second optical fiber link can be connected to WDM receiver to increase the distance depending on a suitable Bit Error Rate (BER).

\section{SIMULATION SETUP}

The setup of the hybrid scheme illustrated in Fig. 1 has been simulated using the OptiSystem version 7 software. The non-hybrid system has also been simulated for comparison with the hybrid system. The MQC code sequence for the 9 users used in the simulation is $(12,4,1)$, while 4 WDM channels $1551.55,1550.75,1549.95$ and 1549.15 are used. The system parameters are as follows; the wavelengths are in C-band for both techniques, the frequency spacing is $100 \mathrm{GHz}$ for the WDM technique, the power transmitted from broadband and laser source are 16 $\mathrm{dBm}$ and $2 \mathrm{dBm}$, respectively, the bandwidth of filters of encoder and decoder for MQC is $0.3 \mathrm{~nm}$, the fiber attenuation is $0.2 \mathrm{~dB} / \mathrm{km}$, the fiber dispersion is 17 $\mathrm{ps} / \mathrm{nm}-\mathrm{km}$, the dispersion slope is $0.075 \mathrm{ps} / \sqrt{\mathrm{km}}$, the external modulator extinction is $30 \mathrm{~dB}$, the bandwidth of notch filter is $0.15 \mathrm{~nm}$, the dark current is $5 \mathrm{nA}$, The thermal noise coefficient of the photo detectors are $1 \times 10^{-22} \mathrm{~W} / \mathrm{Hz}$ and $1.8 \times 10^{-22} \mathrm{~W} / \mathrm{Hz}$ for SAC OCDMA and WDM, respectively, and the data rates used are 622 Mbps and 2.5 Gbps for SAC OCDMA and WDM, respectively. The simulation is kept as real as possible by activating all the attenuation parameters.

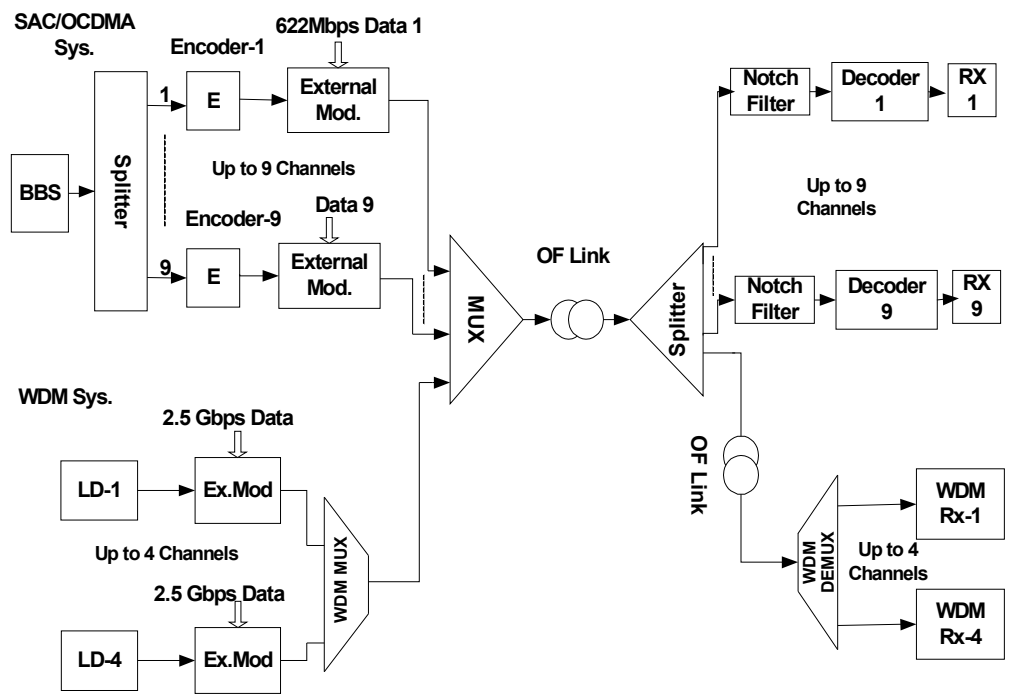

Fig.1 Block-diagram of the hybrid system 
TABLE I

MQC code sequences when $\mathrm{p}=3, \mathrm{~N}=12$, and the selective wavelengths

\begin{tabular}{|c|c|c|c|c|c|c|c|c|c|c|c|c|}
\hline $\mathbf{N}$ & 1 & 2 & 3 & 4 & 5 & 6 & 7 & 8 & 9 & 10 & 11 & 12 \\
\hline Wavelength & 1549 & 1549.3 & 1549.6 & 1549.9 & 1550.2 & 1550.5 & 1550.8 & 1551.1 & 1551.4 & 1551.7 & 1552 & 1551.3 \\
\hline \multirow{9}{*}{ 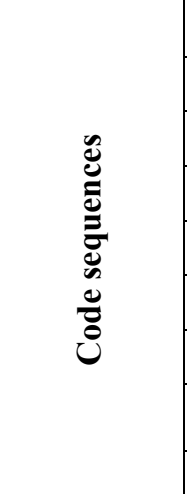 } & 1 & 0 & 0 & 0 & 1 & 0 & 0 & 1 & 0 & 0 & 0 & 1 \\
\hline & 0 & 1 & 0 & 0 & 0 & 1 & 0 & 0 & 1 & 0 & 0 & 1 \\
\hline & 0 & 0 & 1 & 1 & 0 & 0 & 1 & 0 & 0 & 0 & 0 & 1 \\
\hline & 0 & 1 & 0 & 0 & 1 & 0 & 1 & 0 & 0 & 1 & 0 & 0 \\
\hline & 0 & 0 & 1 & 0 & 0 & 1 & 0 & 1 & 0 & 1 & 0 & 0 \\
\hline & 1 & 0 & 0 & 1 & 0 & 0 & 0 & 0 & 1 & 1 & 0 & 0 \\
\hline & 0 & 1 & 0 & 1 & 0 & 0 & 0 & 1 & 0 & 0 & 1 & 0 \\
\hline & 0 & 0 & 1 & 0 & 1 & 0 & 0 & 0 & 1 & 0 & 1 & 0 \\
\hline & 1 & 0 & 0 & 0 & 0 & 1 & 1 & 0 & 0 & 0 & 1 & 0 \\
\hline
\end{tabular}

In the hybrid system, the combined signal is transmitted through the optical fiber link and is split into two parts, one for the receivers of SAC/OCDMA signals, composed of notch filters, match decoders and balance techniques, and the other part is for the receivers of the WDM system, composed of narrowband WDM de-multiplexer to attenuate the broad-band signal that is coming from SAC/OCDMA source, and standard receiver parts of WDM. For the non-hybrid system, each individual system under the same previous parameters was simulated.

Fig. 2 [14] shows the eavesdropper's receiver is based on scanning all selective wavelengths used by SAC/OCDMA. We assume that an eavesdropper knows these wavelengths. The technique of the eavesdropper to detect the code is tapped a coded transmission of an authorized user and performing the calculations to derive the user's entire code word. This is one type of detection processing that needs the amount of time for an eavesdropper to detect the signal and to specify the user's code. However, the eavesdropper does not have the notch filter as the authorized user has. Therefore, the combined overlay signal has passed through the receiver of the eavesdropper.

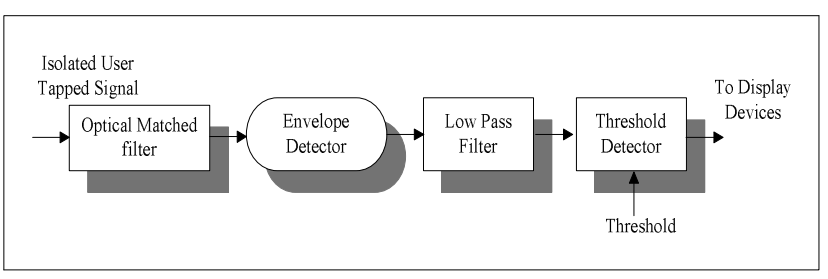

Fig. 2 Eavesdropper's receiver

\section{RESULTS AND DISCUSSION}

The performance of the system is evaluated prior and after hybridization using the Bit Error Rate (BER). The performance of eavesdropper detection is also evaluated using the BER and Q-factor for each optical carrier wavelength of MQC code.

Fig. 3 shows the BER for users of MQC code of SAC/OCDMA with various fiber link distances for hybrid and non-hybrid systems. The BER for hybrid scheme is slightly lesser than the non-hybrid scheme because of the overlapping signals between the SAC/OCDMA and WDM signals. However, the security of the hybrid scheme is higher than the nonhybrid one. This is a logic result and could be illustrated in Table 2 for the eavesdropper performance. All users of MQC of SAC have an approximately equivalent BER when the system is non hybrid, while they have different BERs when the system is hybrid as shown between user 1 and user 2 of MQC in Fig. 3. As some users have more interference by WDM signals than others, we use APD photo-detector for them. This results in a better performance than the normal users who have PIN photo-detector. Fig. 4 shows the BER for user-1 of the hybrid scheme using PIN photo detector and the BER for the same user used APD photo detector. It has been shown in both Fig. 3 and Fig. 4 that both system performance decrease when the distance increases.

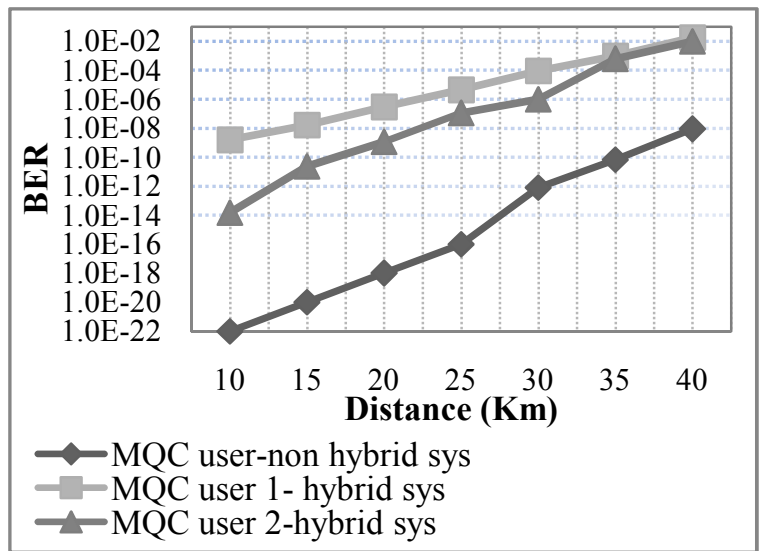

Fig. 3 BER versus distance for MQC users of SAC/OCDMA for hybrid and non-hybrid system 


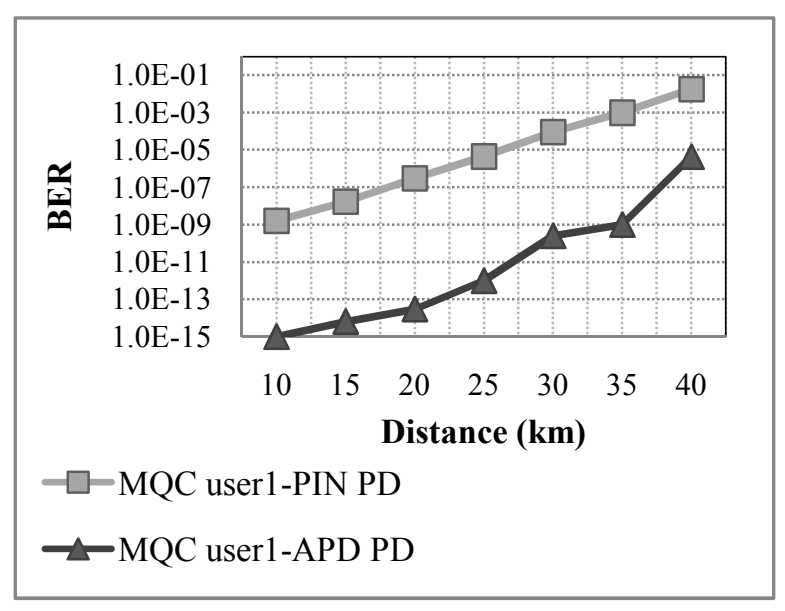

Fig. 4 BER versus distance for MQC user-1used PIN PD and the same user used APD PD

The eye-diagrams in Fig. 5 are obtained when a data rate of $2.5 \mathrm{~Gb} / \mathrm{s}$ is transmitted to (a) a $70 \mathrm{~km}$ for WDM in a hybrid system and to (b) $100 \mathrm{~km}$ for WDM in a non-hybrid system. The BER is 3.9E-9 for (a) and 1.2E12 for (b). The performance for the hybrid system is acceptable and we can get better BER when the distance of fiber link is decreased.

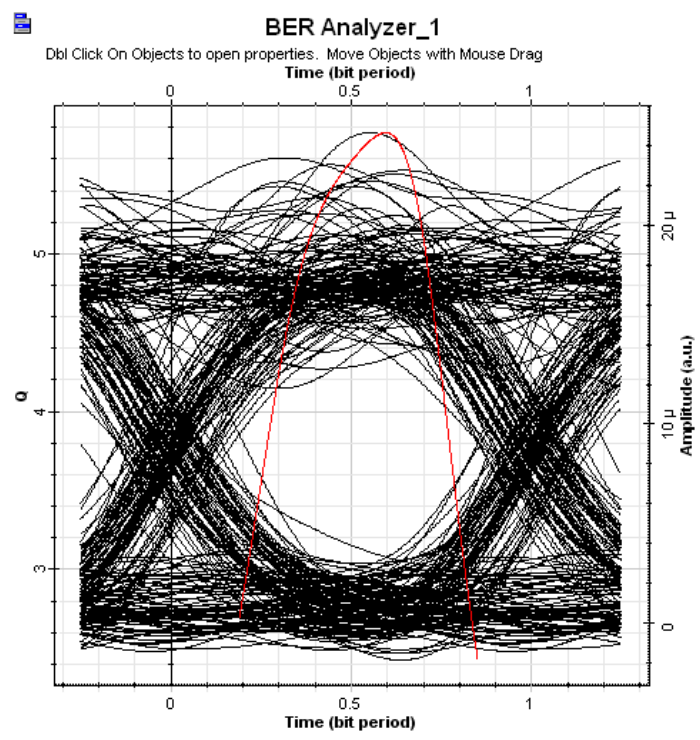

(a)

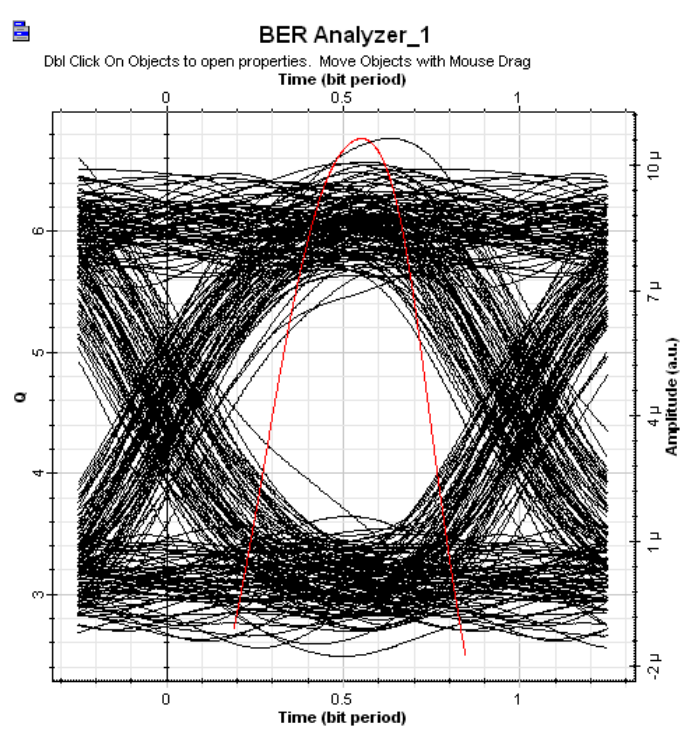

(b)

Fig. 5 Eye diagram for a WDM user, (a) Hybrid scheme with transmission distance of $70 \mathrm{~km}$; (b) Non-hybrid scheme with transmission of distance $100 \mathrm{~km}$

Table 2 shows the results of scanning process for an eavesdropper. The BER and Q-factor for every optical wavelength of MQC code of SAC/OCDMA are illustrated in this table. It has been shown that the difference of values between the hybrid and non-hybrid system is very large. In the non-hybrid system, the Qfactor is more than 9.0, and the BER is lesser than 7.0E21. Therefore, an eavesdropper can easily detect the entire code word with error-free. In comparison with the hybrid system, the range of values differs from 0 to 6.37 for Q-factor and 1 to $4.2 \mathrm{E}-10$ for BER. Therefore, the confusion, and the difficulty for an eavesdropper to intercept and detect the MQC code of SAC/OCDMA is high.

TABLE II

Scanning results for an eavesdropper

\begin{tabular}{|c|c|c|c|c|}
\hline & \multicolumn{2}{|c|}{ Hybrid System } & \multicolumn{2}{c|}{$\begin{array}{c}\text { Non-Hybrid } \\
\text { System }\end{array}$} \\
\hline Wavelength & BER & Q-factor & BER & Q-factor \\
\hline 1549 & $3.73 \mathrm{E}-02$ & 1.75 & $7.10 \mathrm{E}-21$ & 9.3 \\
\hline 1549.3 & 1 & 0 & $5.81 \mathrm{E}-37$ & 12.5 \\
\hline 1549.6 & $3.67 \mathrm{E}-03$ & 2.68 & $6.25 \mathrm{E}-25$ & 10.1 \\
\hline 1549.9 & $3.00 \mathrm{E}-02$ & 1.88 & $8.40 \mathrm{E}-31$ & 11.4 \\
\hline 1550.2 & $2.52 \mathrm{E}-02$ & 1.95 & $5.56 \mathrm{E}-31$ & 11.4 \\
\hline 1550.5 & $3.13 \mathrm{E}-02$ & 1.84 & $1.22 \mathrm{E}-33$ & 11.9 \\
\hline 1550.8 & $4.39 \mathrm{E}-02$ & 1.69 & $3.92 \mathrm{E}-28$ & 10.6 \\
\hline 1551.1 & $4.40 \mathrm{E}-04$ & 3.32 & $7.20 \mathrm{E}-22$ & 9.4 \\
\hline 1551.4 & $2.28 \mathrm{E}-02$ & 1.97 & $4.33 \mathrm{E}-32$ & 11.8 \\
\hline 1551.7 & $2.40 \mathrm{E}-02$ & 1.96 & $1.75 \mathrm{E}-29$ & 10.8 \\
\hline
\end{tabular}




\begin{tabular}{|c|c|c|c|c|}
1552 & $3.35 \mathrm{E}-06$ & 4.21 & $3.70 \mathrm{E}-31$ & 11.5 \\
\hline 1552.3 & $4.20 \mathrm{E}-10$ & 6.37 & $7.10 \mathrm{E}-24$ & 9.5 \\
\hline
\end{tabular}

\section{CONCLUSIONS}

The proposed SAC/OCDMA-WDM overlay scheme with MQC code as the signature address code provides enhanced data confidentiality compared to MQC code of SAC/OCDMA only scheme. The separation process between different techniques is presented by utilizing notch filters. In addition, the design parameters are illustrated for simulation of both hybrid and non-hybrid systems. The performance and enhancing security of the hybrid network is investigated by measuring the biterror-rates (BERs) and comparing the non-hybrid network. APD photo detector is used instead of PIN photo detector to improve the performance of the MQC users who suffer more from the WDM interferes. An enhancement of data networks security can be increased for this system by using long transmitted code and many channels from WDM transmitter.

\section{REFERENCES}

[1] J. Huang, Y. Chang, and C. Hsu, "Hybrid WDM and optical CDMA implemented over waveguide-grating-based fiber-to-thehome networks," Optical Fiber Technol., vol. 13, pp. 215-225, 2007.

[2] Z. Wei, H. M. H. Shalaby, and H. Ghafouri-Shiraz, "Modified quadratic congruence codes for fiber Bragg-grating-based spectral-amplitude coding optical CDMA systems," $J$. Lightwave Technol., vol. 19, pp. 1274-1281, Sept. 2001

[3] S. Galli, R. Menendez, P. Toliver, T. Banwell, J. Jackel, J. Young, and S. Etemad, "Experimental results on the simultaneous transmission of two 2.5 Gbps optical-CDMA channels and a $10 \mathrm{Gbps}$ OOK channel within the same WDM window," in Proc. IEEE Optical Fiber Communication (OFC), Anaheim, CA, Mar. 6-11, 2005, vol. 3, pp. 29-31.

[4] B. B. Wu and E. E. Narimanov, "A method for secure communications over a public fiber optical network," Opt. Express, VOL. 14, pp. 3738-3751, 2006.

[5] R. C. Menendez, P. Toliver, S. Galli, A. Agarwal, T. Banwell, J. Jackel, J. Young, and S. Etemad, "Network Applications of Cascaded Passive Code Translation for WDM-Compatible Spectrally Phase-Encoded Optical CDMA," Journal of Lightwave Technology, VOL. 23, NO. 10, pp. 3219-3231, 2005.

[6] K. Kravtsov, B. Wu, I. Glesk, P. R. Prucnal, and E Narimanov, "Stealth Transmission over a WDM Network with Detection Based on an All-Optical Thresholder," ILEOS annual meeting, WH2, October 2007. pp. 480-481.

[7] C. C. Yang, "Hybrid Wavelength-Division-Multiplexing/ Spectral-Amplitude-Coding Optical CDMA System," IEEE Photonic Technology Letters, VOL. 17, NO. 6, pp. 1343-1345, JUNE 2005.

[8] K. Kitayama, X. Wang, and N. Wada, "OCDMA Over WDM PON-Solution Path to Gigabit-Symmetric FTTH," Journal of Lightwave Technology, VOL. 24, NO. 4, pp. 1654-1662, APRIL 2006.

[9] A. Teixeira1, A. Vieira1, J. Andrade1, A. Quinta1, M. Lima1, R. Nogueira1, P. André1, and G. Beleffi, "Security Issues in
Optical Networks Physical Layer," in Proc. Transparent Optical Networks ICTON, Athens, 2008, pp. 123-126.

[10] A. Mohammed, N.M. Saad, E.I. Babekir, N. Elfadel, S.A.Aljunid, M.S.Anuar.," Modeling and Simulation of the Double Weight Code Family Detection Schemes," in Proc. Allerton Conf. Intelligent and Advanced Systems, Kuala Lumpor, 2007, pp. 348-351.

[11] B. B. Wu, P. R. Prucnal, and E. E. Narimanov., "Secure Transmission Over an Existing Public WDM Lightwave Network," IEEE Photonic Technology Letters, VOL. 18, NO. 17, pp. 1870-1872, 2006

[12] M. P. Fok, K. Kravtsov, Y. Deng, Z. Wang, T. Wang, and P. R. Prucnal, "Providing Network Security with Optical Signal Processing," in Proc. Optical Communications and Networks (ICOCN 2008), Singapore, December 2008. [Invited]

[13] M.J. LaGasse, W. Charczenko, M.C. Hamilton, and S. Thaniyavarn, "Optical carrier filtering for high dynamic range fiber optic links," Electron Letters, VOL.30, (1994), pp. $2157-$ 2158.

[14] Hesham A. B., Sahbudin S., and Mahamod I., "Security Performance of Spectral Amplitude Code OCDMA: Spectrally Encoded Pulse Bandwidth Effects", J. Opt. Commun., VOL. 30, NO.4, (2009), pp. 242-247. 